\title{
On Common Factor and Principal Component Representations of Data: Implications for Theory and for Confirmatory Replications
}

\author{
Keith F. Widaman \\ University of California at Riverside
}

To appear in: Structural Equation Modeling

Accepted Date: 16 May 2018

\begin{abstract}
Author's Note:
Keith F. Widaman, Graduate School of Education, University of California at Riverside. Correspondence concerning this article should be addressed to Keith F. Widaman, Graduate School of Education, University of California, 900 University Drive, Riverside, CA 92521. E-mail: keith.widaman@ucr.edu
\end{abstract}

(C) 2018, Taylor \& Francis. This paper is not the copy of record and my not exactly replicate the final, authoritative version of the article. Please do not copy or cite without author permission. The final article will be available, upon publication, via DOI: $10.1080 / 10705511.2018 .1478730$ 


\section{Abstract}

Common factor analysis (FA) and principal component analysis (PCA) are commonly used to obtain lower-dimensional representations of matrices of correlations among manifest variables. Whereas some experts argue that differences in results from use of FA and PCA are small and relatively unimportant in empirical studies, the fundamental rationales for the two methods are very different. Here, FA and PCA are contrasted on four key issues: the range of possible dimensional loadings, the range of potential correlations among dimensions, the structure of residual covariances and correlations, and the relation between population parameters and the correlational structures with which they are associated. For decades, experts have emphasized indeterminacies of the FA model, particularly indeterminacy of common factor scores. Determinate in most respects, a heretofore unacknowledged, pernicious indeterminacy of PCA is demonstrated: the indeterminacy between PCA structural representations and the correlational structures from which they are derived. Researchers are often advised to use either FA or PCA in exploratory rounds of data analysis to understand and refine the dimensional structure of a domain before moving to structural equation modeling in later theory-testing, confirmatory, replication studies. Results from the current study suggest that PCA is an unreliable method to use for such purposes and may lead to serious misrepresentation of the structure of a domain. Hence, PCA should never be used if the goal is to understand and represent the latent structure of a domain; only FA techniques should be used for this purpose, as only FA provides reliable structural representations as the basis for confirmatory tests in future studies.

Keywords: Common factor analysis, principal component analysis, factor loadings, oblique factors, structural equation modeling 


\section{On Common Factor and Principal Component Representations of Data: Implications for Theory and for Confirmatory Replications}

The first paper on common factor analysis (FA) was published over a century ago (Spearman, 1904), appearing shortly after the initial publication on what became known as principal components analysis (PCA) (Pearson, 1901), and the major contributions describing PCA for the social sciences appeared over 85 years ago (Hotelling, 1933a, 1933b). As befits techniques with broad applicability, FA and PCA have been used in countless publications to represent or account for manifest variables in many domains. Innumerable contributions to and refinements of the two techniques have been made over the ensuing decades, and a good number of comparative arguments regarding the relative advantages of each technique have been offered (Snook \& Gorsuch, 1989; Steiger, 1994; Velicer \& Jackson, 1990; Widaman, 1993).

Both FA and PCA have been touted as methods for representing the structure in a data set. FA and PCA were initially developed as methods for the analysis of correlation matrices, as opposed to covariance matrices, and the rest of this paper will retain this focus on correlation matrices, with no loss in generality. In small correlation matrices, researchers may be able to "see" the patterns in relations among manifest variables. But, as the size of a correlation matrix increases, patterns in the correlation matrix become ever more difficult to discern. As a result, researchers are frequently advised to use FA or PCA to aid in the understanding of the complex patterns of relations among manifest variables, and practicing scientists often do so without a great deal of attention to or inspection of the correlation matrix.

The central thesis of the current paper is that fundamental differences exist between FA and PCA representations of data, and these differences have yet to be explicated fully. The goal of the present work is to illustrate some of these neglected matters, particularly with regard to the 
issue of replication of results across studies, an issue of considerable current interest in the field. Some experts play down differences between FA and PCA results. For example, Goldberg and Velicer (2006), in a chapter offering general advice to personality researchers on analytic choices, argued that differences between the two methods were usually quite minor and that PCA might be preferred because it avoids certain problems of FA, such as improper solutions. As argued below, fundamental problems with PCA representations are sufficient to question seriously the use of PCA for isolating dimensions aligned with latent theoretical constructs.

The issue of differences between FA and PCA is of critical importance for state-of-the-art methods such as confirmatory factor analysis and latent variable structural equation modeling (SEM). Both of the latter techniques are restricted extensions of the exploratory FA model. Researchers are often advised to use exploratory methods, such as FA or PCA, to refine the dimensional structure of their manifest variables before moving to confirmatory factor analysis or SEM to replicate and extend their empirical work. Exploratory FA should provide a reasonable basis for preliminary analyses that lead to later investigations using SEM, but PCA can provide an extremely tenuous basis for later work with SEM, as shown below.

\section{FA versus PCA: Rationales, Models, and Contrasts}

The mathematical models underlying FA and PCA are linear in form, with manifest variables represented as linear functions of latent or summary dimensions. Here, the linear models for each technique are briefly presented. For ease of presentation, but no loss in generality, the one-dimensional case will be emphasized, but multidimensional cases will be used as needed.

\section{FA and Its Linear Model}

Rationale. From its inception, FA has been a mathematical model that emphasized 
implications for understanding both the manifest variables included in the analysis and the latent variables isolated. In the first paper on FA, Spearman (1904) made a strong case for the presence of a single latent dimension of intelligence, which he christened ' $g$ ' for general intelligence, and most of this initial contribution touted the ability of $g$ to account for correlations among manifest variables in the ability domain. Importantly, Spearman marshaled support for his arguments by noting the differential loadings of particular manifest variables on the $g$ factor, with manifest variables that most clearly required more sophisticated cognitive skill having systematically higher loadings than those of a less intellective nature.

Thurstone $(1935,1947)$ continued this tradition of emphasizing both manifest and latent variables in the mental ability domain, even as he expanded the factorial structure of abilities to identify multiple factors he termed Primary Mental Abilities. In many writings, Thurstone and his associates (e.g., Thurstone \& Thurstone, 1941) spent much time attempting to understand the nature of the Primary Mental Abilities and to reconcile multiple-factor theory with Spearman's $g$ theory. In this vein, the development of oblique rotations of primary factors and higher-order factor analysis of correlations among first-order factors offered, in the words of Thurstone and Thurstone (1941), a rapprochement with $g$ theory, with primary mental abilities existing at the level of first-order factors and $g$ at the second-order level.

In his earliest writings, Thurstone emphasized the need for replication across studies of latent variables aligned with primary mental abilities, but insisted that the factorial representation of particular manifest variables was of paramount importance. When discussing factorial invariance, Thurstone wrote:

It is a fundamental criterion of a valid method of isolating primary abilities that the weights of the primary abilities for a test must remain invariant when it is moved from 
one test battery to another test battery. ${ }^{*}$ The same principle can be stated as a fundamental requirement of a successful factorial method, that the factorial description of a test must remain invariant when the test is moved from one battery to another which involves the same common factors. $\dagger$ (Thurstone, 1947, p. 361, emphasis in original). ${ }^{1}$

This quote provides clarity on the issue of the importance of FA yielding crucial information for manifest variables, as invariant relations of latent variables to particular manifest variables are necessary when replicating and extending factorial work. Moreover, for both Thurstone (1947) and Spearman (1904), FA was a method developed to model or represent as accurately as possible the correlations among manifest variables in an efficient, reduced-rank solution in terms of effects of latent variables on the manifest variables in the analysis.

Model. The linear FA model for scores on manifest variables may be written as:

$$
z_{j i}=\lambda_{j 1} \eta_{1 i}+\ldots+\lambda_{j m} \eta_{m i}+\varepsilon_{j i}
$$

where $z_{j i}$ represents the standardized score $(M=0, S D=1)$ of person $i(i=1, \ldots, N)$ on manifest variable $j(j=1, \ldots, p), \lambda_{j \kappa}$ is the loading of manifest variable $j$ on common factor or latent variable $\kappa(\kappa=1, \ldots, m), \eta_{\kappa i}$ is the factor score of individual $i$ on latent variable $\kappa$, and $\varepsilon_{j i}$ is the score of person $i$ on the unique factor for manifest variable $j$.

Equation 1 may be written for all $p$ manifest variables in matrix form as:

$$
\mathbf{Z}=\boldsymbol{\Lambda} \boldsymbol{\eta}+\boldsymbol{\varepsilon}
$$

where $\mathbf{Z}$ is a $(p \times 1)$ vector of scores on the $p$ manifest variables for a given subject, $\boldsymbol{\Lambda}$ is a ( $p \times \mathrm{x}$ $m$ ) matrix of loadings of the $p$ manifest variables on the $m$ latent variables, $\boldsymbol{\eta}$ is an $(m \times 1)$ vector of scores of the given subject on the $m$ latent variables, and $\boldsymbol{\varepsilon}$ is a $(p \times 1)$ vector of unique factor scores. Assuming that the latent variables and unique factors have mean expectations of zero, have unit variances, and are mutually uncorrelated, postmultiplying each side of Equation 2 by 
itself and taking expectations leads to:

$$
\mathbf{R}=\boldsymbol{\Lambda} \Psi \boldsymbol{\Lambda}^{\prime}+\boldsymbol{\Theta}
$$

where $\mathbf{R}$ is the ( $p \times p)$ population matrix of correlations among manifest variables, $\boldsymbol{\Psi}$ is the ( $m$ $\mathrm{x} m$ ) matrix of correlations (or covariances) among latent variables, $\boldsymbol{\Theta}$ is the ( $p \times p$ ) diagonal matrix of unique factor variances, and other symbols were defined above. In the single-factor case, $\boldsymbol{\Lambda}$ is a column vector of loadings of manifest variables on the single latent factor, and $\boldsymbol{\Psi}$ is a unit-valued scalar, so Equation 3 can simplify to $\mathbf{R}=\mathbf{\Lambda} \mathbf{\Lambda}^{\prime}+\boldsymbol{\Theta}$.

\section{PCA and Its Linear Model}

Rationale. The rationale for PCA was, at least at its inception, rather different than that for FA. Indeed, PCA as portrayed by Hotelling (1933a, 1933b) was a method of resolving $p$ correlated manifest variables into $p$ orthogonal dimensions. Then, Hotelling (1935) quickly followed up his initial work by arguing that the first principal component was the most reliable composite of the $p$ manifest variables. It appears that the primary goal of PCA was simply to reexpress the $p$ manifest variables in terms of $p$ components that were orthogonal and conditionally variance maximized. Thus, the component scores, which are weighted linear combinations of manifest variables, were the focus of PCA, and the nature of the manifest variables and their specific weightings were of decidedly less importance.

Model. The full-rank linear PCA model for manifest variables may be written as:

$$
z_{j i}=l_{j 1} w_{1 i}+\ldots+l_{j p} w_{p i}
$$

where $l_{j k}$ is the loading of manifest variable $j$ on component $k(k=1, \ldots, p), w_{k i}$ is the score of individual $i$ on component $k$, and other symbols were defined above.

Equation 4 may be written for all $p$ manifest variables in matrix form as:

$$
\mathbf{Z}=\mathbf{L w}
$$


where $\mathbf{L}$ is a $(p \times p)$ matrix of loadings of the $p$ manifest variables on the $p$ principal components, $\mathbf{w}$ is a ( $p \times 1)$ vector of scores of the given subject on the $p$ components, and other symbols were defined above. Assuming that component scores have mean expectations of zero, have unit variances, and are mutually uncorrelated, postmultiplying each side of Equation 5 by itself and taking expectations leads to:

$$
\mathbf{R}=\mathbf{L L}^{\prime}
$$

where all symbols were defined above. Researchers frequently retain for interpretation only a relatively small number of principal components, so Equation 6 can be rewritten as:

$$
\mathbf{R}=\mathbf{F F}^{\prime}+\mathbf{G G}^{\prime}=\mathbf{F F}^{\prime}+\Delta=\mathbf{F}^{*} \mathbf{C F}^{* \prime}+\Delta
$$

where $\mathbf{F}$ is a $(p \times m)$ matrix of loadings of the $p$ manifest variables on the $m$ principal components retained for interpretation, $\mathbf{G}$ is a $(p \times(p-m))$ matrix of loadings of the $p$ manifest variables on the $(p-m)$ components discarded, $\Delta$ is the $(p \times p)$ non-diagonal matrix of covariances among residuals of the manifest variables $\left(\boldsymbol{\Delta}=\mathbf{G G}^{\prime}\right), \mathbf{F}^{*}$ is the $(p \times m)$ rotated component loading matrix, $\mathbf{C}$ is the $(m \times m)$ matrix of correlations among rotated components, and other symbols were defined above. If more than a single component is retained in $\mathbf{F}$, the components can be rotated orthogonally or obliquely, leading to a representation that is (see the last member of Equation 7) similar to the FA solution in Equation 3, at least on the surface.

\section{Comparisons of FA and PCA}

Goals of FA and PCA. Shortly after Hotelling (1933a, 1933b) published his work on PCA, a flurry of publications contested his approach to factoring the correlation matrix with unities on the diagonal (e.g., Burt, 1937; Kellogg, 1936a, 1936b; Thomson, 1934; Thurstone, 1935). Thurstone noted that his methods conflicted with those of Hotelling in several ways, including (a) retaining a number of dimensions equal to the number of manifest variables, 
thereby failing to obtain a reduced rank representation, (b) failing to rotate dimensions, and (c) using unities on the diagonal in place of estimates of communality. All of these are matters of concern, but of greater concern is the differential underlying goals of the two techniques.

Thomson (1939a, 1939b) offered one of the first and most direct accounts of the differing primary goals of FA and PCA. As Thomson argued, the primary goal of FA is to explain the correlations among the manifest variables. Communality estimates should be used in FA so that correlations among manifest variables can be optimally reproduced by the factor solution. In the course of reproducing the correlations among manifest variables, the FA solution represents or "explains" variance of the manifest variables, and the total variance explained by the $m$-factor solution is the sum of the communalities of the manifest variables. So, in FA, the primary goal is to explain as well as possible the correlations among manifest variables, and explaining variance in manifest variables is accomplished as a byproduct.

In contrast, explained variance is the primary goal of PCA. That is, a reduced-rank, $m$ dimensional component solution yields the maximal amount of total variance in the $p$ manifest variables explained by a reduced-rank solution in $m$ dimensions. Thus, the primary goal of PCA is to explain as much variance as possible in the manifest variables in a small number of dimensions, and reproducing the correlations among manifest variables is accomplished as a byproduct. A full-rank PCA solution will account perfectly for all of the variances of and all of the correlations among manifest variables, but a full-rank PCA solution does not achieve the scientific goal of a reduced-rank representation of relations among manifest variables.

Results using FA and PCA. Most comparisons of results of FA and PCA have centered on values in the loading matrices. That is, interest has typically been restricted to comparisons of FA and PCA loading matrices $\boldsymbol{\Lambda}$ and $\mathbf{F}^{*}$, respectively; researchers could also have compared the 
correlations among rotated dimensions in $\boldsymbol{\Psi}$ and $\mathbf{C}$, respectively, but this has rarely been done (for an exception, see Ogasawara, 2003). In simulation studies, researchers often simulated data with equal factor loadings. For example, if three manifest variables loaded highly on each of three factors, all non-zero factor loadings would be an identical value (e.g., .4 or .8) in a given simulated population. In addition, for reference in the rest of this paper, researchers often used population standardized FA pattern loadings of $.4, .6$, and .8 to reflect low, moderate, and high levels of communality, respectively, and these values have become standard reference values in the field.

One matter that has not received sufficient attention is that all Monte Carlo studies comparing FA and PCA have used a FA method to generate their data and then used FA and PCA to analyze the data. Several approaches can be used to generate such data. For example, one could construct a population correlation matrix from population parameters in a FA model and then draw sample correlation matrices directly from the population correlation matrix. Or, one could simulate individual observations on a given manifest variable by multiplying the population common factor loading by a simulated common factor score and sum the resulting value with the corresponding unique factor loading multiplied by a simulated unique factor score. Or, one could simulate individual observations by computing the product of an $(N \times p)$ matrix of random deviates with $\mathbf{L}^{\prime}$, as advocated by Kaiser and Dickman (1962) and Wherry, Naylor, Wherry, and Falllis (1965). Regardless of the method used, data are simulated in a fashion consistent with FA, and resulting data are subjected to FA and PCA analyses.

The preceding approach to simulating data has led to some odd, but underappreciated findings. For example, assume three manifest variables with population FA loadings of .8. The FA model in Equation 3 will lead to a ( $3 \times 3$ ) population correlation matrix with off-diagonal 
values of .64. If FA is used to analyze this matrix, one unsurprisingly recovers the .8 loadings that generated the correlation structure. If PCA is used, the resulting loadings on the first component are .872, and authors frequently refer to this value as the "population loading" for each of the three manifest variables under PCA (cf. Velicer \& Fava, 1987; Ogasawara, 2003). Suppose one augmented this correlation matrix with three additional manifest variables that also had population FA loadings of .8 , leading to a (6 x 6) matrix with off-diagonal values of .64. Extracting one common factor from this matrix would, again not surprisingly, recover loadings for each manifest variable of .8. But, PCA will lead to a first component with six loadings of .837, and researchers treat these as the "population loadings" under PCA for this matrix. But, this result is contrary to the requirement stated by Thurstone (1947), because the so-called "population value" of the loadings for the first three manifest variables shifted from .872 in the three-variable analysis to .837 in the six-variable analysis. This result supports a contention that there is no single, identifiable population loading for a given manifest variable under PCA, but the "population value" of the PCA loading for a given manifest variable varies as a function of the battery being analyzed. If so, standard errors for PCA loadings have little or no meaning, because they do not refer to sampling variability about a specific population parameter for a given manifest variable.

A motivating example. A one-factor example is shown in Table 1, which contains correlations among 10 variables: three manifest variables with high communality (High1 High3), four with moderate communality (Mod1 - Mod4), and three with low communality (Low1 - Low3). The correlation matrix was formed using the FA factor loadings shown on the right side of the table and Equation 3. Then, the correlation matrix was analyzed twice, once using a FA method (ULS estimation) and once with PCA (principal axes), with results for the 
two methods shown on the right side of Table 1. Under FA, high communality variables have loadings of .8 , moderate communality variables loadings of .6 , and low communality variables loadings of .4. Under PCA, all loadings are higher than FA loadings, with discrepancy between FA and PCA loadings inversely related to level of communality. Note the "population loading" of .658 for Mod4 in the PCA solution. Note as well that all eigenvalues are zero except for the first under FA, but that all 10 eigenvalues are non-zero and are varied for PCA.

Insert Tables 1 and 2 about here

In Table 2, results are shown for smaller batteries selected from the matrix in Table 1. In a given study, a researcher might not select all 10 manifest variables for the latent dimension under study, but might select three- or four-variable batteries. In each of the six scenarios in Table 2, manifest variable Mod4 is included in the battery, but in different batteries Mod4 is paired with two or three high communality variables, two or three moderate communality variables, or two or three low communality variables, as might occur by chance in empirical investigations. For all six scenarios, FA recovered the .6 population loading for Mod4 in each of the 6 scenarios. But, the PCA loadings for Mod4 vary across scenarios as a function of the number and characteristics of the other variables in the analysis. Indeed, the PCA loadings for Mod4 vary in chameleon-like fashion, being highest when the battery includes high communality variables and lowest when the battery includes low communality indicators. In addition, holding communality of the other variables in the battery constant, all PCA loadings are higher in threevariable batteries than in four-variable batteries. Further, all PCA loadings for Mod4 in the six scenarios in Table 2 are larger than the so-called "population value" of .658 shown in Table 1. 
These results in Tables 1 and 2 underscore the problematic concept of a population loading for any particular manifest variable under PCA. Stated differently, the population loading for a particular manifest variable on a given dimension under PCA is a misnomer or fiction (i.e., a "category mistake" in the sense of Ryle, 1949); instead the so-called "population loading" for a given variable under PCA will vary as a function of the number of additional indicators for the dimension and the levels of correlation among these variables included in a battery.

\section{Population Structures Consistent with the FA Model}

In this section, some basic aspects of population structures under the FA model will be discussed, allowing informative comparisons with PCA structures in the following section.

\section{Range of FA Loadings}

Consider the one-factor case in which correlations follow the positive manifold typically seen for ability variables, so that correlations among manifest variables can vary between 0 and 1 (i.e., no negative correlations). This stipulation does not harm generality, because factors with bipolar loadings (e.g., positive loadings for extraversion items and negative loadings for introversion, or reverse worded, items) can achieve positive manifold of correlations among indicators if negatively-oriented indicators are reverse scored.

Assuming positive manifold of correlations among manifest variables, the potential range of FA loadings is from 0 to 1 , with loadings of 0 if off-diagonal correlations are zero ranging up to loadings of 1 if off-diagonal values are unity. Admittedly, off-diagonal correlations will never attain values of unity in empirical data, but this is the theoretical upper bound for these elements, just as zero is the lower bound in a positive manifold pattern. If off-diagonal values are not equal (as in Table 1), FA loadings still can range from 0 to 1 , but will vary as a function of level of correlation of a given manifest variable with other manifest variables. 
In the multiple factor case, similar observations hold, with loadings between 0.0 and +1.0. If the positive manifold of correlations holds among manifest variables, the resulting FA structure can have a very simple pattern. For example, each latent variable can have a number of manifest variables with positive, non-zero loadings, remaining manifest variables with loadings of zero, and a positive manifold of correlations among latent variables.

\section{Range of Correlations among Rotated Common Factors}

Correlations among oblique common factors can vary between -1.0 and +1.0 . If a positive manifold pattern holds for correlations among manifest variables, then correlations among oblique common factors would be expected to vary between 0 and +1.0 . In empirical studies, correlations among the Big 5 measures of personality are often quite low (i.e., near zero), but correlations among ability factors can often be quite high (e.g., in a range from .50 to .70). In simulation studies, various levels of correlation can be and have been entertained. For example, correlations between factors that near unity (e.g., .95) might be useful in a study of improper solutions (van Driel, 1978) and associated testing of constrained estimation in such situations (Stoel, Garre, Dolan, \& van den Wittenboer, 2006).

\section{Correlations among Unique Factors}

As is clear from Equation 3, the population correlations among unique factors in the FA model are zero. Even if these correlations are zero in the population, they will not be zero in any sample from the population (cf. MacCallum \& Tucker, 1991). However, in theory, in the population, and in the bowels of a computer simulating data from a specified FA structure, the population covariances and correlations among unique factors are zero under the FA model, and they are constrained to zero in any application of exploratory FA.

\section{Relations of FA Parameters with Population Correlations}


In population correlation matrices constructed using Equation 3, a 1:1 relation holds between population FA loadings and the resulting population correlation matrix. That is, given a specified set of common factor loadings in $\boldsymbol{\Lambda}$, use of Equation 3 will lead to one and only one correlation matrix. Conversely, if a population correlation matrix has been constructed according to Equation 3, FA estimation with optimal estimates of communality will lead to one and only one FA solution. This claim must be qualified if the solution contains more than one common factor, as rotational indeterminacy of the common factor solution means that multiple orientations of the axes (e.g., orthogonal, oblique) can appear rather different. However, if an independent cluster solution with multiple factors is used to construct the correlation matrix, optimal FA methods will recover precisely this generating solution, including both the loadings on factors and the correlations among optimally rotated factors. Furthermore, this population structure is developed by specifying a small number of parameters: $p$ factor loadings, $m(m-1)$ correlations among oblique factors, and $p$ unique factor variances (with the $p$ unique variances constrained by other estimates to yield unit diagonal elements in a correlation metric). Given this small number of parameters, Equation 3 can be used to compute the population correlation matrix consistent with FA parameters.

The relations between FA loadings and factor intercorrelations and the correlations among manifest variables with which they are related has become commonplace to users of SEM. If latent variable variances are fixed at unity, the correlation between two indicators of a given factor is reproduced by the product of their respective standardized factor loadings. The correlation between two indicators of different factors will be reproduced by the triple product of the two relevant factor loadings and the correlation between the two latent variables. Thus, researchers have come to expect that, if a common factor model has close fit to data, the 
correlations among manifest variables will be closely recovered by relatively simple multiplicative functions of factor loadings and correlations among factors.

\section{Population Structures Consistent with PCA}

The results in Tables 1 and 2 and the preceding discussion of population structures consistent with the FA model may strike some as moot. That is, if one constructs population correlation matrices consistent with the FA model, it is not surprising that FA can recover the model parameters that generated the correlational structure and not surprising that PCA may do a poorer job of recovering the population loadings. The common reaction might be, "So what? If one generated data from a correlation matrix consistent with a specified PCA loading structure, then PCA would undoubtedly do a better job of recovering those loadings than would FA." Although this is true, essentially no work has been done to date on generating correlation structures consistent with specified PCA loading patterns, a task to which we now turn.

\section{Range of PCA Loadings}

Under the positive manifold correlation pattern assumed above, the potential range of PCA loadings is rather more restricted than the range of FA loadings. Because the first principal component must have an eigenvalue greater than or equal to 1, loadings on the first component must average $\sqrt{1 / a}$ or higher, where $a$ is the number of manifest variables loading on the component. Thus, if one used PCA to analyze a 3 x 3 matrix and obtained three loadings of .6, these should not be construed as moderate-sized loadings, but as extremely small loadings. If one used PCA to analyze a 3 x 3 identity matrix, one would obtain three loadings of $\sqrt{1 / 3}=.5774$, and loadings of .6 fall barely above this minimal value. Indeed, PCA loadings of .6 are obtained from a $3 \times 3$ correlation matrix with off-diagonal correlations of only .04, or manifest variables that have virtually nil relations with one another. With 4 manifest variables, PCA loadings must 
average .50 or higher (i.e., PCA of a 4 x 4 identity matrix will yield a first component with equal loadings of .50); with 10 manifest variables, PCA loadings must average .316 or higher; and with 25 manifest variables, PCA loadings must average .20 or higher. Thus, the potential range of PCA loadings does increase as the number of indicators per dimension increases, but this increase in range is rather slow and remains problematic in most applications. A crucial note is that the above results are based on the number of manifest variables that load on each given (rotated) component, not on the total number of manifest variables in the analysis.

\section{Range of Correlations among Rotated Principal Components}

As with loadings discussed above, the range of correlations among optimally rotated principal components is also much more restricted than comparable values from FA. Unfortunately, a simple formula for describing the range of correlations among oblique components that is applicable in general is not available. The range of correlations among components is discussed below in connection with equal component loadings, but unequal population component loadings lead to structures too complex for simple and useful formulas for the range of correlations among rotated components.

\section{Correlations among Residuals}

In contrast to FA, the residual structure in PCA is not diagonal and cannot be diagonal, because the matrix $\boldsymbol{\Delta}\left(=\mathbf{G G}^{\prime}\right)$ in Equation 7 is of order $p$, but has a rank of $(p-m)$. Given its deficient rank, non-zero off-diagonals must be found in $\Delta$. To my knowledge, no useful general discussion of the pattern of these residual covariances has ever been provided in the $85+$ years since PCA was first discussed in the social sciences. Indeed, residual covariances and correlations among manifest variables have rarely been discussed in treatments of PCA and are rarely, if ever, included as standard output by computer programs. As will be demonstrated, the 
patterns of correlations among residuals in PCA often appear haphazard in pattern and difficult or impossible to describe or justify.

\section{Relations of PCA Parameters with Population Correlations}

In a preceding section, a claim was made that a 1:1 relation holds between a FA model with specified parameters and the correlation matrix generated by Equation 3. For PCA, the 1:1 relation holds only for 3 x 3 correlation matrices. Even here, some 3 x 3 correlation matrices associated with specified PCA loadings will appear bizarre. Moreover, once one moves to situations with 4 or more manifest variables loading on a single component, a 1:n relation (with $n$ very large or even infinite) holds between specified loadings on a first principal component and correlation matrices that will reproduce the specified PCA loadings. This is a heretofore unacknowledged indeterminacy of PCA, an indeterminacy that threatens the use of PCA for understanding and interpreting the structure of a domain.

The reasons for the foregoing claims are as follows: In a full-rank PCA solution, one can specify particular loadings on the first component, but one must then estimate or specify (in some fashion) the $p(p-1)$ loadings on remaining components. The full-rank PCA model invokes $p(p+1) / 2$ constraints: (a) $p(p-1) / 2$ orthogonality constraints, such that $\mathbf{L}$ 'L is diagonal with eigenvalues on its diagonal; and (b) $p$ variance constraints, such that $\operatorname{diag}\left(\mathbf{L} \mathbf{L}^{\prime}\right)=\mathbf{I}$. With 3 manifest variables, six loadings on the two discarded components in $\mathbf{G}$ must be estimated, and the $3(3-1) / 2=3$ orthogonality constraints and 3 variance constraints result in 6 equations to use in calculating the 6 unknown loadings in $\mathbf{G}$. But, with 4 manifest variables, the $4(4-1) / 2=6$ orthogonality constraints plus 4 variance constraints lead to 10 equations, but 12 loadings in $\mathbf{G}$ must be calculated, so the solution is indeterminate, having more unknowns than equations. One can impose two or more additional constraints to identify the solution, but these constraints are 
arbitrary, and choice of different constraints will lead to different correlation matrices, hence the 1:n relation between specified loadings and potential correlation matrices.

Equal PCA loadings. If equal PCA component loading values are specified and if one invokes the additional (unnecessary) constraint that all off-diagonal correlations must be equal, then it is possible to construct one-dimensional correlation matrices with any number of manifest variables that lead to prescribed PCA loadings. It is possible to show that the off-diagonal correlation $r$ that achieves this goal can be calculated as:

$$
r=l^{2}-\frac{1-l^{2}}{p-1}
$$

where $l$ is the desired PCA loading and other symbols were defined above. For example, if a first component from a 3 x 3 matrix should have equal loadings of .6, then Equation 8 shows that offdiagonal correlations should be $r=.6^{2}-\left(1-.6^{2}\right) /(3-1)=.04$. If the first component from a $4 \mathrm{x}$ 4 matrix should have equal PCA loadings of .6, the off-diagonal correlations should be $r=.6^{2}-\left(1-.6^{2}\right) /(4-1)=.1467$. Moreover, solving Equation 8 for the PCA loading $l$ leads to:

$$
l=\sqrt{\frac{(p-1) r+1}{p}}
$$

where all symbols were defined above. Hence, knowing the number of manifest variables and the uniform off-diagonal correlations, Equation 9 yields the PCA loadings that result. But, having equal PCA loadings does not require equal off-diagonal correlations so Equations 8 and 9 no longer apply and no general formulas are available if the assumption of equal off-diagonal correlations is relaxed. Furthermore, with four or more manifest variables, if the "equal offdiagonal correlations" stipulation is relaxed, an infinite number of different correlation matrices can be found that yield equal loadings on the first component. 
If two components each have equal specified loadings, it is possible to develop a correlation matrix that will recover a specific correlation between rotated components. The reproduced correlation between two vectors $x$ and $y$ can be expressed as:

$$
r_{x y}=h_{x} h_{y} \operatorname{Cos}_{x y}
$$

where $r_{x y}$ is the correlation between $x$ and $y, h_{x}$ and $h_{y}$ are the lengths of the vectors for $x$ and $y$, and $\operatorname{Cos}_{x y}$ is the cosine of the angle between $x$ and $y$, where this cosine is the desired correlation between the oblique components If one wished to have a PCA solution with three manifest variables with loadings of .8 on Component 1,3 manifest variables with loadings of .8 on Component 2 , and a .50 correlation between the oblique components, the following calculations would achieve this goal: Equation 8 would lead to $r=\left(.8^{2}-1\right) /(3-1)+.8^{2}=.46$, which would be the correlations among indicators for a given component. If one desired the correlation between components to be .5 , use of Equation 10 would lead to $\sqrt{.46} \sqrt{.46}(.50)=.23$, so the manifest variables loading on Component 1 should correlate .23 with manifest variables loading on Component 2, although this may present problems, as shown below.

Unequal PCA loadings. With three manifest variables, unique 3 x 3 correlation matrices can be obtained that will produce specified unequal loadings when subjected to PCA. However, with four or more manifest variables, an infinite number of correlation matrices can produce specified unequal PCA loadings. In a unidimensional solution, if the first PCA eigenvalue is identified as EV1, EV1 must be greater than or equal to unity. Further, the remaining $(p-1)$ eigenvalues must each be less than 1.0 (or else the structure would not be unidimensional) and must sum to ( $p-\mathrm{EV} 1)$, and an infinite set of sets of eigenvalues satisfy the latter requirement. Because each unique set of eigenvalues will lead to a different correlation matrix, an infinite 
number of correlation matrices is associated with any PCA solution with four or more indicators.

\section{Examples of Prescribed FA and PCA Structures}

In this section, various examples of FA and PCA structures are provided to illustrate claims made above. When performing FA, unweighted least squares (ULS) estimation was used, and standard principal axes extraction was used for PCA. For two-dimensional solutions, HarrisKaiser orthoblique rotations were employed with power of 0.0. All analyses were performed with SAS; all programs are available on request.

\section{Population Correlations Based on FA}

One-factor populations. In the top of Table 3, Scenario 1 depicts a one-factor solution for three manifest variables with equal FA loadings of .60. The off-diagonal correlations among manifest variables were all .36 , FA recovered the population loadings of .60 perfectly, and the off-diagonal residual covariances (below the diagonal) and correlations (above the diagonal) from this FA solution were all zero. For PCA, equal loadings of .757 were obtained, consistent with Equation 9. As shown in Table 3, the residual covariances under PCA were all -0.21 and residual correlations were all -0.50 .

Insert Table 3 about here

In Scenario 2, the three manifest variables had FA loadings of $.80, .60$, and .40, so varied but averaged the same as loadings under Scenario 1. FA recovered population loadings perfectly, and off-diagonal residual covariances and correlations were again zero. The PCA loadings varied as one might expect, but note that manifest variable 2, which had equal FA loadings of .60 under both Scenario 1 and 2, had differing PCA loadings of .757 and .782 in Scenarios 1 and 2, 
respectively. Further, the PCA residual covariances and correlations, while still uniformly negative, varied in a seemingly haphazard fashion. Interestingly, the residual covariances averaged -0.21 and residual correlations averaged -0.50 , identical to values in Scenario 1.

Scenario 3 in Table 3 is identical to Scenario 1 except for the addition of a fourth variable with a population FA loading of .60. As with Scenario 1, Scenario 3 revealed uniform .36 correlations among manifest variables; FA recovered population loadings perfectly, and residual covariances and correlations were all zero. Use of PCA led to uniform loadings of .721, consistent with Equation 9. All residual covariances were -.16 (cf. values of -.21 for Scenario 1), and all residual correlations were -.33 (cf. values of -.50 for Scenario 1).

The final section of Table 3 shows results for Scenario 4, which is similar to Scenario 2 except for addition of a fourth manifest variable with a population loadings of .60. Thus, in Scenario 4, population FA loadings varied, but averaged .60 (similar to Scenario 2). As with previous scenarios, FA recovered the population FA loadings perfectly, and all residual covariances and correlations were zero. Under PCA, loadings varied and exhibited positive bias in inverse relation to FA loading magnitude. Residual covariances once again varied in a haphazard fashion, and residual correlations were all -.32 except for the -.40 between the two variables that had middling population FA loadings of .60. PCA residual covariances varied but averaged the same as under Scenario 3, and the same occurred for residual correlations. Proponents of PCA should provide some explanation or justification for these results.

One finding should be noted. Manifest variable 2 had population FA loadings of .60 in all four scenarios, and FA recovered these values precisely. However, under PCA estimation, the PCA loadings for manifest variable 2 were never the same across scenarios. This finding shows that PCA of data consistent with a FA population structure fails to satisfy Thurstone's dictum 
that the factorial description of a manifest variable must remain constant when the variable is moved from one battery to another that contains the same latent variables.

Two-factor populations. Two alternative two-factor FA population structures are shown in Table 4, which shows the population correlations, FA loadings, and correlation between the two factors. Residual correlations and covariances are given in Table 5. Scenario 5 involved two factors having an independent cluster solution, with three variables loading .60 on Factor 1, three variables loading .60 on Factor 2, and a .50 correlation between the factors. Each factor therefore has a loading structure that was identical to that for Scenario 1 (i.e., equal loadings of .60), but two correlated factors were now present. Use of FA techniques reproduced population loadings and the factor correlation perfectly. As shown in Table 5, all residual correlations and covariances were zero for the FA solution.

When PCA was applied to the correlation matrix for Scenario 5, many results were similar to those for Scenario 1. Specifically, the three factor loadings on each component were .757, within-component residual covariances were uniformly -.21, and within-component residual correlations were all -.50, all identical to values for Scenario 1 (see Table 3). Somewhat surprisingly, all between-component residual covariances and correlations (i.e., of variables $1-3$ with variables $4-6$ ) were all precisely zero (see Table 5) as were the non-defining loadings on each component. Finally, the overestimation of loadings was accompanied by underestimation of the component correlation (.314), as predicted by Equation 10 .

Insert Tables 4 and 5 about here

Scenario 6 was constructed to be similar to Scenario 2 with varied FA loadings of .80, 
.60 , and .40 (averaging .60) on each factor, but now with two factors that were correlated .50 . Analyzing the correlation matrix in Table 4 using FA led to exact reproduction of factor loadings and the correlation between the two factors, and all unique factor covariances and correlations were exactly zero. Applying PCA to the correlation matrix led to loadings shown in Table 4. Oddly, despite the within-component identity of varied factor loadings between Scenarios 2 and 6, the Scenario 6 loadings differed markedly from comparable values in Scenario 2, with loadings that were much more homogeneous in Scenario 6 (ranging from .771 to .709) than in Scenario 2 (which ranged from .825 to .642). The non-defining loadings on the two components now differed from zero (they were exactly zero in Scenario 5), but averaged approximately zero, as expected under optimal oblique rotation. Finally, the residual covariances and correlations exhibited a new and different pattern (as shown in Table 5). All of these covariances and correlations were non-zero, and several of these values were of positive valence. The largest of these values were for the within-component residual covariances and correlations, and the between-component residual covariances and correlations appeared to average zero, despite varying in haphazard fashion.

\section{Population Correlations based on PCA}

As noted above, if correlations among manifest variables were generated to be consistent with a prescribed PCA structure, then PCA should recover the "generating" parameters well and FA should yield less optimal results. In this section, this proposition will be evaluated.

One-component populations. Examples of data consistent with a PCA structure will illustrate additional differences between FA and PCA structures, and all of these examples are shown in Table 6. The first section of Table 6 is for Scenario 7, with three manifest variables that should each load .80 on the first principal component. Note that all correlations among manifest 
variables were .46. Applying PCA to this correlation matrix indeed perfectly reproduced the population PCA loadings of .80, and the PCA residual covariances and correlations had expected form (e.g., residual correlations uniformly -.50, identical to Scenario 1). As expected, FA of the correlation matrix led to lower estimated factor loadings (.678), but the residual covariances and correlations were all zero, as in Scenarios 1 through 6, implying that a one-factor FA solution was acceptable for this correlation matrix. Thus, PCA recovered the specified component loadings perfectly, and FA did not.

Insert Table 6 about here

Scenario 8 is one with varied population PCA loadings of $.80, .70$, and .60 , which led to the correlation matrix shown in Table 6. PCA of this correlation matrix perfectly reproduced the population PCA loadings. The PCA residual covariances and correlations were no longer uniform in magnitude, but the residual correlations averaged -.50 as they had in Scenario 7. When FA methods were applied to this correlation matrix, the highest loading (.846) was surprisingly overestimated, whereas the remaining two loading were severely underestimated. The FA residual covariances and correlations were all exactly zero, as expected.

The final three-variable scenario shown in Table 6 is Scenario 9, in which the three manifest variables load $.80, .60$, and .40 on the first principal component, identical to the loadings on the first principal factor in Scenario 2 (see Table 3). The resulting correlation matrix, shown in Table 6 , has a rather odd appearance, with all correlations being rather low $(\leq|.14|)$ and one of them negative in sign. A negative correlation between two manifest variables that both load positively on a dimension is difficult, if not impossible, to explain; few, if any, proponents 
of PCA would have predicted that this could occur. Still, PCA of the correlation matrix reproduced the population loadings perfectly. The PCA residual covariances and correlations had a strikingly haphazard pattern, although the residual correlations appeared to average approximately -.50, as they had in Scenarios 7 and 8. The upshot for Scenario 9 is that PCA appears to yield a viable solution, with one manifest variable loading highly, one moderately, and one relatively low on the first principal component.

FA applied to the correlation matrix for Scenario 9 resulted in a Heywood case, given the single negative correlation in the $3 \times 3$ correlation matrix. It is well known that $3 \times 3$ correlation matrices with either 0 or 2 negative correlations below the diagonal can be well fit by a FA solution, but $3 \times 3$ matrices with 1 or 3 negative correlations below the diagonal cannot be well fit using FA. So, the Heywood case would be expected. Invoking an upper bound of 1.0 on the first factor loading led to the results in Table 6. FA clearly provided a very poor set of estimates of factor loadings. Notably, the FA residual covariance and correlation matrices now were not diagonal, being unable to yield perfect fit in one dimension. A proponent of FA might tout these results as favoring FA, because the results indicate a serious problem for the data set, a problem masked by the seemingly acceptable PCA solution for this matrix.

Turning to four-variable problems, consider first Scenario 10, in which all four manifest variables load .80 on the first principal component. The first component has an eigenvalue of 2.56 (or $4 \cdot .8^{2}$ ), so the remaining three eigenvalues must sum to 1.44 (or $4-2.56$ ). If the indeterminacy of the PCA model in this scenario is resolved by fixing the remaining three eigenvalues to be equal (0.48), one arrives at the correlation matrix for Scenario 10, shown in Table 6. PCA of this matrix reproduced the four .80 loadings on the first principal component, and the residual covariances and correlations had expected patterns. Because off-diagonal 
correlations were uniformly .52, FA of this matrix led to a well-fitting one-factor solution with lower loadings of .721, and off-diagonal residual covariances and correlations that were zero.

However, the eigenvalues of discarded components in $\mathbf{G}$ need not be equal. Adding 0.24 to the first of these discarded components and subtracting 0.24 from the third resulted in eigenvalues of $0.72,0.48$, and 0.24 , which sum to 1.44 (as required) and led to Scenario 11 . PCA of the Scenario 11 correlation matrix recovered the four .80 loadings on the first principal component, but the residual covariances and correlations had a rather odd appearance, with two zero values, two moderate negative values, and two large negative values. FA applied to the correlation matrix and restricting the solution to one factor again led to a factor with uniform loadings of .721. The residual covariances and correlations for the FA model had patterns that were as odd as the pattern for the PCA. However, the non-diagonal form of the matrices of FA residual covariances and correlations implied that this correlation matrix did not conform well to a one-factor representation, suggesting one dimension is not a proper FA solution.

Arbitrarily fixing the eigenvalues of discarded components in $\mathbf{G}$ to $0.80,0.32$, and 0.32 (which again sum to 1.44 , as required) led to Scenario 12, which had a correlation structure that was rather different than those for Scenarios 10 and 11. PCA of this matrix again led to a first principal component with four loadings of .80 . The PCA residual covariances and correlations exhibited a different, but odd pattern, with some positive values and some negative values. Using FA to extract a one-factor solution for this matrix again led to a single factor with loadings of .721. As with Scenario 11, the non-diagonal form of the FA residual covariances and correlations implied that a one-dimensional FA solution for this data set is not appropriate.

A brief summary of results across Scenarios 10 through 12 is in order. The three correlation matrices look rather different, yet these are simply three of the infinite number of 
different correlation matrices that are consistent with four loadings of .80 on the first principal component. PCAs of the three matrices provide perfect component loading invariance across scenarios of the component solution, with four loadings of .80 on the first component. For each scenario, only a single component has an eigenvalue over 1.0, implying a one-dimensional solution was adequate in each case. If these three correlation matrices were from different samples and PCA were applied to each, failure to inspect the correlation matrices would lead one to conclude, mistakenly, that the structure of the data had been replicated perfectly, whereas the correlation matrices clearly belie this conclusion.

Some might argue that FA results also misleadingly support a conclusion of replication for Scenarios 10 through 12 because, for each of these three scenarios, FA led to a first factor with uniform loadings of .721. However, the FA residual structures tell an important story. The FA for Scenario 10 led to diagonal matrices of residual covariances and correlations, suggesting that one factor was an adequate representation of these data. In contrast, despite the identical first factor loadings, the matrices of FA residual covariances and correlations for Scenarios 11 and 12 imply that one-dimensional FA solutions do not provide a perfect representation of these data, arguing against perfect replication of results across these three scenarios. For both Scenario 11 and 12, FA suggests that more than one dimension is needed, contrasting with the satisfactory "fit" (i.e., one component with eigenvalue > 1) of a one-component solution under PCA. The final two scenarios shown in Table 6, Scenarios 13 and 14, are merely examples of what can occur with unequal loadings of four manifest variables on a single component. The inequality of loadings specified was minor, with the first three variables loading .80 and the fourth variable loading .60 on the first component. The first component had an eigenvalue of 2.28, so the remaining three eigenvalues sum necessarily to 1.72 . One arbitrary selection of three 
eigenvalues led to Scenario 13, and a different arbitrary choice led to Scenario 14. The two correlation matrices appear very different: the correlation between variables 1 and 2 is rather lower in Scenario $13(.55)$ than in Scenario $14(.65)$, and the correlation between variables 1 and 4 is decidedly higher in Scenario 13 (.40) than in Scenario 14 (.21). Still, PCA applied to each of these matrices led to a single component with eigenvalue above 1.0 and recovered precisely the specified population PCA loadings. Consistent with the differing patterns of correlation, the matrices of residual PCA covariances and correlations were strikingly different across scenarios.

FA applied to the correlation matrices for Scenarios 13 and 14 led to results that differed less than one might have expected. For each scenario, the non-diagonal matrices of residual covariances and correlations clearly implied that a one-factor solution was inadequate for both of these scenarios, indicating failure to replicate FA structures across these scenarios.

Two-component populations. Two-dimensional component populations, particularly with unequal loadings, are so woefully underdetermined and therefore indeterminate that such populations will be discussed only briefly here. A previous section laid out an argument that correlations among optimally rotated components were subject to potentially major restrictions of range, and the nature of this restriction is discussed here. With positive manifold of correlations among manifest variables, common factors can correlate between 0 and 1.0. Such is not the case with principal components. Table 7 lists the maximal possible correlation between two optimally rotated principal components as a function of the number of indicators per component and the magnitude of component loadings, assuming equal loading in a given solution. For example, if four manifest variables load .60 on the first component and four manifest variables load .60 on the second component, the highest possible correlation between rotated components is only .30. If a correlation structure is generated with a correlation between 
components higher than .30 with these loadings, the second eigenvalue will fall below 1.0 , and a researcher would almost certainly conclude that a single-component solution was adequate for this data set with eight manifest variables. Inspection of Table 7 shows that component loadings must be rather high and/or the number of indicators per component must be rather high for a correlation between rotated components as high as .60 to be found in a PCA solution. Correlations of .60 or .70 are often found between oblique common factors in the mental ability domain, so use of FA and PCA of ability data would likely lead to very different results with regard to correlations among rotated dimensions. This issue attains added importance given the fact that correlations among oblique dimensions are the basis for higher-order FA or PCA.

Insert Table 7 about here

\section{Discussion}

FA and PCA are used so frequently in empirical studies that most practicing scientists have little or no appreciation for the fundamental differences in the goals of the two procedures or of the potential differences in results that might be obtained when using the two techniques. Restricted or "confirmatory" forms of FA, using programs for structural equation modeling (SEM), are used increasingly to test theoretical ideas about the factor structure of different domains. Despite the widespread use of exploratory versions of FA and PCA and confirmatory forms of FA, the relations and distinctions among these methods have rarely been highlighted.

\section{FA and PCA Representations of Data}

One principal goal of the current paper was to compare and contrast FA and PCA with regard to four key aspects of their representations of data. These four aspects of representations 
involve (a) the magnitude of loadings, (b) the magnitude of relations among oblique dimensions, (c) the structure of residual covariances and correlations, and (d) the relations between parameter values and the correlations among manifest variables with which they are associated. Simulation studies of FA and PCA have often used loadings of $.80, .60$, and .40 to denote relatively high, medium, and low levels of communality, and data are then simulated based on these values using FA methods. Once data are simulated, FA and PCA methods are applied to the data, and results are compared across methods of analysis. The high, medium, and low loading values have been used so frequently that they have been more-or-less commonly accepted as standards when evaluating empirical studies. So, certain general principles about analytic results may be commonly accepted, but others are almost surely underappreciated.

Let us consider in turn the four key aspects of representations of data outline above first, the magnitude of loadings. When using exploratory or confirmatory FA, researchers readily expect that common factor loadings, even in one-factor solutions, can vary between 0 and 1.0 in absolute magnitude. Indeed, if correlations among manifest variables are very close to zero, researchers assume that factor loadings would be approximately zero, and they would be correct in this surmise. This does not hold when PCA is used. With uniform off-diagonal elements, PCA loadings can never reach zero. If one analyzed a 25-variable identity matrix (with off-diagonal correlations of zero) with FA, one would obtain a factor matrix with all 25 variables loading zero on the first common factor. PCA would yield a first principal component with loadings of .20 for all 25 variables. Indeed, PCA of a 100-variable identity matrix would yield a first component with loadings of .10 for all 100 variables, so PCA loadings approach zero very slowly and never get to zero. Commonly used values of $.8, .6$, and .4 loadings may well represent high, medium, and low FA loadings, but values that constitute high, medium, and low PCA loadings are unclear 
and have never been stated with any certainty or theoretical justification by proponents of PCA.

Next, the range of possible correlations among oblique dimensions is a worthy topic of discussion. Researchers almost surely assume that correlations between oblique common factors can vary between 0 and 1.0 in absolute magnitude, and this again is a correct assumption when FA is used. But, again, this assumption is false for PCA. As shown in Table 7, the maximal correlation between rotated components is often rather low and depends on both the number of variables loading on components and the magnitude of component loadings. The correlation between obliquely rotated principal components is probably a very poor predictor of the magnitude of correlation between latent variables unless the true factor loadings are extremely high and the number of indicators is also very large.

The third aspect of representations concerns the residual covariances and correlations remaining after extracting the optimal number of factors or components. When using confirmatory FA or SEM with multiple-indicator models, researchers readily expect unique factors to be uncorrelated, and this is the same assumption made in exploratory FA. Then, in longitudinal modeling, covariances among unique factors for the same manifest variable across time are often required, consistent with the theory that unique factors consist of a combination of specific (i.e., reliable) and error variance - and that the specific variance in indicators may be correlated across time. In short, substantive psychometric theory dictates reasonable residual structures in FA.

In contrast, PCA implicitly invokes the assumption that residual covariances and correlations are not zero, but no attempt is typically made either to admit or emphasize this point. Because residual covariances and correlations after PCA are rarely, if ever, examined, there is no need to admit their existence. However, these residual covariances and correlations are part of 
the PCA "model," so deserve attention. Furthermore, the haphazard nature of the patterns in these residual covariances and correlations often seems to defy explanation, which may contribute to deciding to ignore these values. As a final note here, experts in structural modeling have long derided the inclusion of non-hypothesized covariances among unique factors in confirmatory FA, terming such estimates "garbage parameters" that capitalize on chance, are usually identified through use of large modification indices, and are included only to improve model fit. But, garbage parameters of this sort abound when PCA is used to analyze data but cause no consternation to data analysts, who are probably unaware of the existence of the residual covariances and the need to justify them.

The fourth and final aspect of FA and PCA representations is the relation between parameter values and correlational patterns with which they are associated, and this may be the most important message of this manuscript. The use of various indices of model fit in SEM, such as the standardized root mean square correlation (SRMR), has attuned researchers to the issue of residual covariances and correlations in SEM and the goodness of fit of models to data. If the SRMR for a given model is very small (e.g., <.02), this means that the covariance structure of the manifest variables is closely reproduced by the model. That is, one could use model estimates to reproduce the covariances among manifest variables and could use the standardized estimates to reconstruct the correlations among manifest variables with considerable precision. This has long been a concern for FA, because the primary, driving goal of FA is to represent closely the correlations (or covariances) among manifest variables.

The primary goal of PCA is to explain variance in manifest variables, not their correlations or covariances. As a result, PCA often provides a rather poor representation of the correlations among manifest variables. Moreover, as shown in Scenarios 10 through 12, a 
particular PCA representation can be consistent with very different correlation matrices. Thus, knowing the loadings of manifest variables on the first principal component provides almost no information with regard to the likely correlations among those manifest variables. This is a pernicious indeterminacy of PCA - the lack of any clear, definable relation between component loadings and the structure of the correlations among manifest variables from which they were derived. Given this indeterminacy, the utility and interpretability of PCA loadings is unclear at a fundamental substantive level.

\section{Generating FA and PCA Data Structures}

Simulation studies of FA and PCA use one of several different methods to generate simulated data, but all of these methods are based on fundamental aspects of the mathematical theory underlying FA. Both population and sample correlation matrices from a specified FA structure can easily be constructed. Analyzing the population correlation matrix with FA should, unsurprisingly, recover the FA parameter estimates used in constructing the matrix. Analyzing the population correlation matrix with PCA leads to parameter values that are termed the population PCA loadings for the data, even though these values are simply the output from analyses, not a priori values based on mathematical theory underlying PCA.

One contribution of this paper is the recognition that one can generate data from specifiable PCA structures and that this requires a very different approach than for generating data from specified FA structures. For the one-dimensional case, unique population correlation matrices for PCA structures with 3 manifest variables with equal or unequal loadings can be constructed, although some of these matrices have undesirable characteristics (cf. Scenario 9 in Table 6). With four manifest variables, the indeterminacies in the PCA formulation makes arbitrary constraints necessary, and different arbitrary decisions lead to different correlation 
matrices for the same specified set of component loadings. With five or more manifest variables loading on a single component, the indeterminacies in the relations between PCA loadings and the correlations from which they are derived are so great that no useful general treatment is possible. Still, the approach needed to construct correlation matrices that yield specified PCA loadings is useful to consider, particularly the difficulties that increase dramatically as the number of manifest variables increases.

\section{Implications for Theory}

The findings reported here have implications for both mathematical and substantive theory. One implication is a reinforcement of the centrality of population correlations and associated loadings in theorizing about the structure of a domain. Basic to FA theory is the concept of a matrix of population correlations (or covariances) among manifest variables. If one had access to the population correlation matrix and fit the optimal model to the data, one would obtain the population factorial description of each and every manifest variable. For example, Scenario 1 (Table 3) is an example of three manifest variables loading .60 on a common factor. When an additional variable loading .6 on the factor was added, leading to Scenario 3 , the correlations among the first three variables were unaltered as were the loadings of the first three variables on the common factor extracted. Similar stability of correlations among manifest variables and their related factor loadings occurs even if loadings vary (see Scenarios 2 and 4 in Table 3).

These findings of stability do not hold for PCA. For three variables to load .80 on the first principal component, uniform off-diagonal population correlations must be .46 (Scenario 7, Table 6). If one additional manifest variable is added to this three-variable problem that also should load .80 on the first principal component and if one requires uniform off-diagonal 
correlations, the correlations would now have to be .52 (Scenario 10, Table 6). Thus, to retain specific population component loadings, the population correlations among the first three manifest variables would have to differ depending on whether three or four manifest variables were included in the analysis. This implies that the concept of a constant population correlation matrix for particular manifest variables with specified PCA loadings cannot be maintained in PCA, so just what PCA loadings mean at a fundamental level is open to question. This indeterminacy of relations between population correlations and PCA loadings was illustrated in Tables 1 and 2 for manifest variable Mod4. Holding the population correlations in Table 1 constant, the component loading for Mod 4 for the 10-variable analysis was .658, but the loadings for Mod4 jumped around between .717 and .771 in Table 2 depending on the composition (i.e., the number of manifest variables and their levels of communality) of selected batteries in which it was included. This was a brute force demonstration that the notion of a single, identifiable population loading for a particular manifest variable has no meaning or interpretation under PCA.

A second, related implication of current results for mathematical theory concerns standard errors (SEs) of FA and PCA loadings. The concept of a population loading for a given manifest variable is well defined under FA. When sampling observations from a population, the SE for a particular FA loading has a clear meaning, referring to the standard deviation of the sampling distribution of the factor loading. Further, the associated confidence interval (CI) provides information about likely values of the population FA loading: in the long run, 95 of every 100 times a 95\% CI is computed for a given factor loading, the CI will contain the population parameter. No such interpretation is possible for SEs of PCA loadings. For over three decades, SEs of principal component loadings have been propounded (e.g., Ogasawara, 1982, 
1983). But, the associated $95 \%$ CI for a PCA loading has no relation to a population PCA parameter value because no such parameter exists, even in theory. The $95 \%$ CI for a PCA loading has a much more limited interpretation, providing information regarding likely sampleto-sample fluctuation in the PCA loading of that manifest variable provided that nothing about the battery of manifest variables - principally the number of manifest variables loading on each component, their loading levels, and the number of dimensions - changes. If any of these characteristics of the battery of measures changes, the so-called "population PCA loading" would change, so the $95 \%$ CI value would be centered around another, fairly arbitrary PCA loading.

Implications for substantive theory should also be mentioned. First, as discussed above, FA was developed as a theory about manifest variables and the latent variables they reflect. A successful FA should provide empirical evidence for one or more common latent variables and each of the manifest variables in the analysis. As noted above, Thurstone (e.g., 1947) always emphasized that factor analysis should be a method of informing about the factorial composition of manifest variables, that the factorial description of a manifest variable should not change when the manifest variable was moved from one battery to another that assessed the same latent variables. In the process of informing about manifest variables, FA would also inform researchers about the latent variables underlying those manifest variables. Moreover, the substantive goal of FA was the promotion of a science of replication in specifiable domains, with researchers urged to study manifest variables in "replication and extension" studies to verify that the factorial composition of manifest variables could indeed be replicated. No such substantive theory has ever been provided for PCA. Instead, PCA was proposed as a mathematical method to produce linear combinations of manifest variables that had useful properties, such as 
orthogonality and conditional variance maximization. Satisfying these desirable mathematical properties in a given sample virtually ensures the sample-specific, non-replicable nature of the resulting estimates. Thus, whereas FA was developed as a method to utilze in careful replication work, PCA allows no theoretical basis that would support replication.

Second, substantive ideas have been pursued recently through the development of short forms of instruments. One example concerns measures of the Big 5 dimensions of personality, which include Extroversion, Conscientiousness, Agreeableness, Neuroticism, and Openness. The initial version of the Big Five Inventory (BFI) was a 44-item instrument with 8 to 10 items for each of the five personality factors assessed. During the past decade and more, short forms such as the Ten Item Personality Inventory (TIPI), with 2 items per factor - have been developed to shorten the administration time and allow inclusion of measures of the five personality dimensions within larger investigations. Having only 2 items per dimension is precisely the situation in which positive bias in loadings and negative bias in correlations among dimensions would be most apparent if PCA were used in analyses. Unfortunately, little detail is provided in many articles on the TIPI, so it is usually impossible to determine whether FA or PCA methods were used. If PCA were used for these analyses, the results might well be an extremely poor predictor of what the results would be if exploratory or confirmatory FA procedures were used. Use of PCA with short forms of any instrument should be discouraged if the goal is to represent the latent variable structure of the domain.

\section{Implications for SEM}

A final issue for consideration is the use of an analytic technique $-\mathrm{FA}$ or PCA - in exploratory work prior to confirmatory work using SEM. Experts in factor analysis have long recommended that several rounds of exploratory analyses be conducted prior to employing 
confirmatory FA or SEM. This recommendation has been based on decades of experience showing that manifest variables often load on factors in ways unforeseen by investigators, underscoring the need to replicate empirical factor structures. Because of this, researchers are urged to engage in several rounds of exploratory analyses to identify a replicable structure for a domain, which can then be used as the basis for clear a priori specification of models in confirmatory FA and SEM. The quotes from Thurstone $(1935,1947)$ cited earlier demonstrate his commitment to developing factorial methods that emphasize the centrality of replication, using language that is remarkably prescient with regard to current views on solving the current "replication crisis" in psychology and the social sciences.

FA is an exploratory method that is an ideal approach in initial investigations into the dimensionality of a domain. Closely replicating the common factor structure of a domain across multiple samples - with close fit of models to data - implies that the fundamental structure of the domain has been closely replicated. Because the mathematical model underlying exploratory FA is the same model as that underlying confirmatory FA, several rounds of successful replication with exploratory FA should provide a strong basis for predicting the nature a model would take under a confirmatory method of analysis.

In contrast, as several scenarios in the current study showed, PCA does not enable a researcher to have confidence with regard to replication of fundamental structural aspects of results across studies. With three manifest variables loading on a component, seemingly mundane and interpretable PCA solutions can belie odd and unacceptable patterns of correlations among manifest variables. Then, with four or more indicators loading on a component, perfect or near-perfect replication of component loadings across studies can be obtained in situations in which the correlational structure of the manifest variables is not well replicated at all. This is the 
heretofore unackowledged fundamental and pernicious indeterminacy of PCA: that, with four or more manifest variables loading on a component, a specifiable set of component loadings can be recovered from an infinite number of different correlation matrices, matrices that may be rather different from one another. With the current zeitgeist emphasizing the importance of replication of results across studies, essentially perfect replication of PCA loadings across studies can occur even though the fundamental correlational relations among manifest variables is not replicated to any discernable degree. Thus, replication of PCA loadings across studies should never be used as a strong basis for specification of confirmatory FA models.

\section{Limitations}

At least three potential limitations of the current work should be noted. The first limitation is the fact that only population parameter values and population correlation matrices were examined, without related discussion of variation under sampling. Further work could certainly be pursued of sampling effects on parameter estimates under FA and PCA. However, the goal of this project was to discuss the "population" values of certain statistics under FA and PCA, so concentration on population loadings and correlations seemed the most expeditious way to investigate these issues.

A second potential limitation is the use of FA models and PCA parameters that fit perfectly in the population. Introduction of population misfit has become quite common in simulation studies of FA and SEM, and this is valuable as it yields results that are more likely to generalize to results with empirical data. The goal of the present study, however, was to demonstrate the different outcomes of FA and PCA applied to population data that had specifiable "perfect fit" under FA or PCA, fit that was unclouded in any way by misfit in the population. As with the absence of consideration of sampling, the failure to include model misfit 
in the population in the current work is a strength of the current efforts, which were to provide simplified demonstrations of key results.

A third potential limitation was the use of correlation matrices in all examples, rather than covariance matrices. This was done because PCA is uniformly performed on correlation matrices, and exploratory forms of FA are also usually applied to correlation matrices. However, given the relations between correlation and covariance matrices, the conclusions with regard to FA parameters can easily be extended to covariance metrics. Such is not the case for PCA, offering yet another advantage of FA over PCA.

\section{Concluding Remarks}

Over seven decades ago, Thurstone $(1935,1947)$ wrote in terms that have a very contemporary ring, emphasizing the need to develop and promote analytic methods that have the greatest likelihood of replication of results across studies. The results of this current study offer clear demonstrations that FA achieves this goal much better than does PCA, which by comparison produces results that are almost hopelessly sample specific. Many comparisons of FA and PCA have been published for 75 years and more, often generating more heat than light. The current comparative examination of these techniques, while perhaps engendering a bit of heat, hopefully has shed new light on several matters that have been too little discussed or entirely unacknowledged in the past. One principal goal of science is replicability of results, and one path toward that goal in the dimensionalizing of behavioral domains now seems clearer, the path associated with FA. 


\section{References}

Burt, C. (1937). Methods of factor analysis with and without successive approximations. British Journal of Educational Psychology, 7, 172-195. DOI: http://dx.doi.org/10.1111/j.2044-8279.1937.tb03166.x

Driel, O. P. van. (1978). On various causes of improper solutions in maximum likelihood factor analysis Psychometrika, 43, 225-243. DOI: http://dx.doi.org/10.1007/BF02293865

Goldberg, L. R., \& Velicer, W. F. (2006). Principles of exploratory factor analysis. In S. Strack (Ed.), Differentiating normal and abnormal personality ( $2^{\text {nd }}$ ed., pp. 209-237). New York: Springer. Document URL: http://search.proquest.com/docview/621339739?accountid=14521

Hotelling, H. (1933a). Analysis of a complex of statistical variables into principal components. Journal of Educational Psychology, 24, 417-441. DOI: http://dx.doi.org/10.1037/h0071325

Hotelling, H. (1933b). Analysis of a complex of statistical variables into principal components. Journal of Educational Psychology, 24, 498-520. DOI: http://dx.doi.org/10.1037/h0070888

Hotelling, H. (1935). The most predictable criterion. Journal of Educational Psychology, 26, 139-142. DOI: http://dx.doi.org/10.1037/h0058165

Kaiser, H. F., \& Dickman, K. (1962). Sample and population score matrices and sample correlation matrices from an arbitrary population correlation matrix Psychometrika, 27, 179-182. DOI: http://dx.doi.org/10.1007/BF02289635

Kellogg, C. (1936a). The problem of principal components: Derivation of Hotelling's method from Thurstone's. Journal of Educational Psychology, 27, 512-520. DOI: 
http://dx.doi.org/10.1037/h0058774

Kellogg, C. (1936b). The problem of principal components: II - The argument for communalities. Journal of Educational Psychology, 27, 581-590. DOI: http://dx.doi.org/10.1037/h0054809

MacCallum, R. C., \& Tucker, L. R. (1991). Representing sources of error in the common-factor model: Implications for theory and practice. Psychological Bulletin, 109, 502-511. DOI: http://dx.doi.org/10.1037/0033-2909.109.3.502

Ogasawara, H. (2002). Concise formulas for the standard errors of component loading estimates. Psychometrika, 67, 289-297. DOI: http://dx.doi.org/10.1007/BF02294847

Ogasawara, H. (2003). Oblique factors and components with independent clusters. Psychometrika, 68, 299-321. DOI: http://dx.doi.org/10.1007/BF02294803

Pearson, K. (1901). On lines and planes of closest fit to systems of points in space. Philosophical Magazine, 2, 559-572. DOI: https://doi.org/10.1080/14786440109462720

Ryle, G. (1949). The concept of mind. Chicago: University of Chicago Press.

Snook, S. C., \& Gorsuch, R. L. (1989). Component analysis versus common factor analysis: A Monte Carlo study. Psychological Bulletin, 106, 148-154. DOI: http://dx.doi.org/10.1037/0033-2909.106.1.148

Spearman, C. (1904). 'General intelligence,” objectively determined and measured. The American Journal of Psychology, 15, 201-293. DOI: http://dx.doi.org/10.2307/1412107

Steiger, J. H. (1994). Factor analysis in the 1980's and 1990's: Some old debates and some new developments. In I. Borg \& P. P. Mohler (Eds.), Trends and perspectives in empirical social research (pp. 201-224). Berlin: Walter de Gruyter.

Stoel, R. D., Garre, F. G., Dolan, C., \& Wittenboer, G. van den. (2006). On the likelihood ratio 
test in structural equation modeling when parameters are subject to boundary constraints. Psychological Methods, 11, 439-455. DOI: http://dx.doi.org/10.1037/1082$\underline{989 X .11 .4 .439}$

Thomson, G. H. (1934). Hotelling's method modified to give Spearman's g. Journal of Educational Psychology, 25, 366-374. DOI: http://dx.doi.org/10.1037/h0072648

Thomson, G. H. (1939a). The factorial analysis of ability: I. The present position and the problems confronting us. British Journal of Psychology, 30, 71-77. Document URL: http://search.proquest.com/docview/615094011?accountid=14521

Thomson, G. H. (1939b). The factorial analysis of ability. Agreement and disagreement in factor analysis: A summing up. British Journal of Psychology, 30, 105-108. Document URL: http://search.proquest.com/docview/615095381?accountid=14521

Thurstone, L. L. (1935). The vectors of mind. Chicago: University of Chicago Press.

Thurstone, L. L. (1947). Multiple factor analysis. Chicago: University of Chicago Press.

Thurstone, L. L., \& Thurstone, T. G. (1941). Factorial studies of intelligence. Psychometric Monographs, No. 2. Document URL: http://search.proquest.com/docview/615105769?accountid=14521

Velicer, W. F., \& Fava, J. L. (1987). An evaluation of the effects of variable sampling on component, image, and factor analysis. Multivariate Behavioral Research, 22, 193-209. DOI: http://dx.doi.org/10.1207/s15327906mbr2202_4

Velicer, W. F., \& Jackson, D. N. (1990). Component analysis versus common factor analysis: Some issues in selecting an appropriate procedure. Multivariate Behavioral Research, 25, 1-28. DOI: http://dx.doi.org/10.1207/s15327906mbr2501_1

Wherry, R. J., Naylor, J. C., Wherry, R. J., Jr., \& Fallis, R. F. (1965). Generating multiple 
samples of multivariate data with arbitrary population parameters. Psychometrika, 30, 303-314. DOI: http://dx.doi.org/10.1007/BF02289493

Widaman, K. F. (1993). Common factor analysis versus principal component analysis:

Differential bias in representing model parameters? Multivariate Behavioral Research,

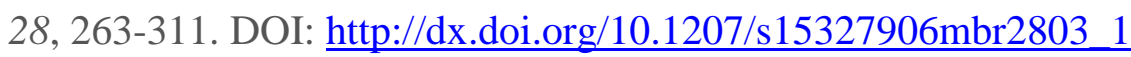




\section{Footnotes}

${ }^{1}$ As noted, the quoted material is from Thurstone (1947, p. 361). The asterisk (*) in the quote referred readers to Thurstone $(1935$, p. 55) and the dagger $(\dagger)$ to Thurstone $(1935$, p. 120) where the italicized parts of the quote, with slight modifications, were first presented. 
Table 1

Population Correlations among 10 Manifest Variables and Full-Battery Common Factor and Principal Component Solutions

\begin{tabular}{|c|c|c|c|c|c|c|c|c|c|c|c|c|}
\hline \multirow[b]{2}{*}{ Variable } & \multicolumn{10}{|c|}{ Correlations } & \multicolumn{2}{|c|}{ Loadings } \\
\hline & High1 & High2 & High3 & Mod1 & Mod2 & Mod3 & Mod4 & Low1 & Low2 & Low3 & FA & PCA \\
\hline High1 & 1.00 & & & & & & & & & & .800 & .816 \\
\hline High2 & .64 & 1.00 & & & & & & & & & .800 & .816 \\
\hline High3 & .64 & .64 & 1.00 & & & & & & & & .800 & .816 \\
\hline Mod1 & .48 & .48 & .48 & 1.00 & & & & & & & .600 & .658 \\
\hline Mod2 & .48 & .48 & .48 & .36 & 1.00 & & & & & & .600 & .658 \\
\hline Mod3 & .48 & .48 & .48 & .36 & .36 & 1.00 & & & & & .600 & .658 \\
\hline Mod4 & .48 & .48 & .48 & .36 & .36 & .36 & 1.00 & & & & .600 & .658 \\
\hline Low1 & .32 & .32 & .32 & .24 & .24 & .24 & .24 & 1.00 & & & .400 & .463 \\
\hline Low2 & .32 & .32 & .32 & .24 & .24 & .24 & .24 & .16 & 1.00 & & .400 & .463 \\
\hline Low3 & .32 & .32 & .32 & .24 & .24 & .24 & .24 & .16 & .16 & 1.00 & .400 & .463 \\
\hline \multicolumn{13}{|c|}{ Eigenvalues } \\
\hline EV1 & & & & & & & & & & & 3.80 & 4.37 \\
\hline EV2 & & & & & & & & & & & .00 & .84 \\
\hline EV3 & & & & & & & & & & & .00 & .84 \\
\hline EV4 & & & & & & & & & & & .00 & .80 \\
\hline
\end{tabular}

Note: To save space, only the first 4 eigenvalues (EVs) for the two solutions are shown; the remaining 6 eigenvalues for the common factor (CF) solution were all .00, and the remaining 6 eigenvalues for the principal component (PC) solution were .64, .64, .64, .51, .36 , and .36. Boldfaced loadings are emphasized to enable easier comparisons with values in Table 2. 
Table 2

Common Factor and Principal Component Solutions for Test Batteries Selected from Manifest Variables in Table 1

\begin{tabular}{|c|c|c|c|c|c|c|c|c|c|c|c|c|}
\hline \multirow[b]{3}{*}{ Variable } & \multicolumn{4}{|c|}{ High communality } & \multicolumn{4}{|c|}{ Moderate communality } & \multicolumn{4}{|c|}{ Low communality } \\
\hline & \multicolumn{2}{|c|}{2 High } & \multicolumn{2}{|c|}{3 High } & \multicolumn{2}{|c|}{2 Moderate } & \multicolumn{2}{|c|}{3 Moderate } & \multicolumn{2}{|c|}{2 Low } & \multicolumn{2}{|c|}{3 Low } \\
\hline & FA & PCA & FA & PCA & FA & PCA & FA & PCA & FA & PCA & FA & PCA \\
\hline High1 & .80 & .859 & .80 & .849 & -- & -- & -- & -- & -- & -- & -- & -- \\
\hline High2 & .80 & .859 & .80 & .849 & -- & -- & -- & -- & -- & -- & -- & -- \\
\hline High3 & -- & -- & .80 & .849 & -- & -- & -- & -- & -- & -- & -- & -- \\
\hline Mod1 & -- & -- & -- & -- & .60 & .757 & .60 & .721 & -- & -- & -- & -- \\
\hline Mod2 & -- & -- & -- & -- & .60 & .757 & .60 & .721 & -- & -- & -- & -- \\
\hline Mod3 & -- & -- & -- & -- & -- & -- & .60 & .721 & -- & -- & -- & -- \\
\hline Mod4 & .60 & .771 & .60 & .724 & .60 & .757 & .60 & .721 & .60 & .742 & .60 & .717 \\
\hline Low1 & -- & -- & -- & -- & -- & -- & -- & -- & .40 & .663 & .40 & .603 \\
\hline Low2 & -- & -- & -- & -- & -- & -- & -- & -- & .40 & .663 & .40 & .603 \\
\hline Low3 & -- & -- & -- & -- & -- & -- & -- & -- & -- & -- & .40 & .603 \\
\hline \multicolumn{13}{|c|}{ Eigenvalues } \\
\hline EV1 & 1.64 & 2.07 & 2.28 & 2.69 & 1.08 & 1.72 & 1.44 & 2.08 & .68 & 1.43 & .84 & 1.61 \\
\hline EV2 & .00 & .57 & .00 & .59 & .00 & .64 & .00 & .64 & .00 & .84 & .00 & .84 \\
\hline EV3 & .00 & .36 & .00 & .36 & .00 & .64 & .00 & .64 & .00 & .73 & .00 & .84 \\
\hline EV4 & & & .00 & .36 & & & .00 & .64 & & & .00 & .71 \\
\hline
\end{tabular}

Note: The terms "High, moderate, and low communality" refer to the population loadings for additional indicators selected to be paired with Mod4 to form a battery of measures. FA = common factor analysis, PCA = principal component analysis. EV1 - EV4 refer to eigenvalues 1 through 4. 
Table 3

Common Factor and Principal Component Solutions for One-Dimensional Batteries Consistent with FA Model

\begin{tabular}{|c|c|c|c|c|c|c|c|c|c|c|c|c|c|c|}
\hline Variable & \multicolumn{4}{|c|}{ Correlations } & \multicolumn{2}{|c|}{ Loadings } & \multicolumn{4}{|c|}{ FA residual structure } & \multicolumn{4}{|c|}{ PCA residual structure } \\
\hline \multicolumn{15}{|c|}{ Scenario 1: 3 manifest variables, equal loadings of .6} \\
\hline Var1 & 1.00 & & & & .60 & .757 & .64 & .00 & .00 & & .43 & -.50 & -.50 & \\
\hline Var2 & .36 & 1.00 & & & .60 & .757 & .00 & .64 & .00 & & -.21 & .43 & -.50 & \\
\hline Var3 & .36 & .36 & 1.00 & & .60 & .757 & .00 & .00 & .64 & & -.21 & -.21 & .43 & \\
\hline Var1 & 1.00 & & & & .80 & .825 & .36 & .00 & .00 & & .32 & -.47 & -.48 & \\
\hline Var2 & .48 & 1.00 & & & .60 & .782 & .00 & .64 & .00 & & -.16 & .39 & -.55 & \\
\hline Var3 & .32 & .24 & 1.00 & & .40 & .642 & .00 & .00 & .84 & & -.21 & -.26 & .59 & \\
\hline \multicolumn{15}{|c|}{ Scenario 3: 4 manifest variables, equal loadings of .6 } \\
\hline Var3 & .36 & .36 & 1.00 & & .60 & .721 & .00 & .00 & .64 & .00 & -.16 & -.16 & .48 & -.33 \\
\hline Var4 & .36 & .36 & .36 & 1.00 & .60 & .721 & .00 & .00 & .00 & .64 & -.16 & -.16 & -.16 & .48 \\
\hline \multicolumn{15}{|c|}{ Scenario 4: 4 manifest variables, varied loadings averaging .6 } \\
\hline Var1 & 1.00 & & & & .80 & .821 & .36 & .00 & .00 & .00 & .33 & -.32 & -.32 & -.32 \\
\hline Var2 & .48 & 1.00 & & & .60 & .736 & .00 & .64 & .00 & .00 & -.12 & .46 & -.32 & -.40 \\
\hline Var3 & .32 & .24 & 1.00 & & .40 & .569 & .00 & .00 & .84 & .00 & -.15 & -.18 & .66 & -.32 \\
\hline Var4 & .48 & .36 & .24 & 1.00 & .60 & .736 & .00 & .00 & .00 & .64 & -.12 & -.18 & -.18 & .46 \\
\hline
\end{tabular}

Note: For FA and PCA residual structures, residual covariances are shown on and below diagonal; covariances rescaled to correlation metric above diagonal (and italicized to ease comparisons). 
Table 4

Common Factor and Principal Component Solutions for Two-Dimensional Batteries Consistent with FA Model

\begin{tabular}{|c|c|c|c|c|c|c|c|c|c|c|c|}
\hline \multirow[b]{2}{*}{ Variable } & \multicolumn{6}{|c|}{ Correlations } & & \multicolumn{2}{|c|}{ FA Solution } & \multicolumn{2}{|c|}{ PCA solution } \\
\hline & Var1 & Var2 & Var3 & Var4 & Var5 & Var6 & & Factor 1 & Factor 2 & Comp. 1 & Comp. 2 \\
\hline \multicolumn{12}{|c|}{ Scenario 5: 6 manifest variables, equal loadings of .6 , factor correlation $=.50$} \\
\hline Var1 & 1.00 & & & & & & & 600 & .00 & .757 & .00 \\
\hline Var2 & .36 & 1.00 & & & & & & .600 & .00 & .757 & .00 \\
\hline Var3 & .36 & .36 & 1.00 & & & & & .600 & .00 & .757 & .00 \\
\hline Var4 & .18 & .18 & .18 & 1.00 & & & & .00 & .600 & .00 & .757 \\
\hline Var5 & .18 & .18 & .18 & .36 & 1.00 & & & .00 & .600 & .00 & .757 \\
\hline \multirow[t]{3}{*}{ Var6 } & .18 & .18 & .18 & .36 & .36 & 1.00 & & .00 & .600 & .00 & .757 \\
\hline & & & & & & & Factor 1 & 1.00 & & 1.000 & \\
\hline & & & & & & & Factor 2 & .50 & 1.00 & .314 & 1.000 \\
\hline \multicolumn{12}{|c|}{ Scenario 6: 6 manifest variables, varied loadings averaging .6 , factor correlation $=.50$} \\
\hline Var1 & 1.00 & & & & & & & .800 & .00 & .771 & .109 \\
\hline Var2 & .48 & 1.00 & & & & & & 600 & .00 & .769 & 004 \\
\hline Var3 & .32 & .24 & 1.00 & & & & & .400 & .00 & .709 & -.122 \\
\hline Var4 & .32 & .24 & .16 & 1.00 & & & & .00 & .800 & .109 & .771 \\
\hline Var5 & .24 & .18 & .12 & .48 & 1.00 & & & .00 & .600 & .004 & .769 \\
\hline \multirow[t]{3}{*}{ Var6 } & .16 & .12 & .08 & .32 & .24 & 1.00 & & .00 & .400 & -.122 & .709 \\
\hline & & & & & & & Factor 1 & 1.00 & & 1.000 & \\
\hline & & & & & & & Factor 2 & .50 & 1.00 & .325 & 1.000 \\
\hline
\end{tabular}


Table 5

Residual Structures for Common Factor and Principal Component Solutions for Two-Dimensional Batteries Consistent with FA Model

\begin{tabular}{|c|c|c|c|c|c|c|c|c|c|c|c|c|}
\hline \multirow[b]{2}{*}{ Variable } & \multicolumn{6}{|c|}{ FA Solution } & \multicolumn{6}{|c|}{ PCA solution } \\
\hline & Var1 & Var2 & Var3 & Var4 & Var5 & Var6 & Var1 & Var2 & Var3 & Var4 & Var5 & Var6 \\
\hline \multicolumn{13}{|c|}{ Scenario 5: 6 manifest variables, equal loadings of .6 , factor correlation $=.50$} \\
\hline Var1 & .64 & .00 & .00 & .00 & .00 & .00 & .43 & -.50 & -.50 & .00 & .00 & .00 \\
\hline Var2 & .00 & .64 & .00 & .00 & .00 & .00 & -.21 & .43 & -.50 & .00 & .00 & .00 \\
\hline Var3 & .00 & .00 & .64 & .00 & .00 & .00 & -.21 & -.21 & .43 & .00 & .00 & .00 \\
\hline Var4 & .00 & .00 & .00 & .64 & .00 & .00 & .00 & .00 & .00 & .43 & -.50 & -.50 \\
\hline Var5 & .00 & .00 & .00 & .00 & .64 & .00 & .00 & .00 & .00 & -.21 & .43 & -.50 \\
\hline Var6 & .00 & .00 & .00 & .00 & .00 & .64 & .00 & .00 & .00 & -.21 & -.21 & .43 \\
\hline \multicolumn{13}{|c|}{ Scenario 6: 6 manifest variables, varied loadings averaging .6 , factor correlation $=.50$} \\
\hline Var1 & .36 & .00 & .00 & .00 & .00 & .00 & .34 & -.38 & -.49 & -.13 & -.11 & .01 \\
\hline Var2 & .00 & .64 & .00 & .00 & .00 & .00 & -.14 & .41 & -.59 & -.11 & -.04 & .08 \\
\hline Var3 & .00 & .00 & .84 & .00 & .00 & .00 & -.21 & -.28 & .54 & .01 & .08 & .16 \\
\hline Var4 & .00 & .00 & .00 & .36 & .00 & .00 & -.04 & -.04 & .00 & .34 & -.38 & -.49 \\
\hline Var5 & .00 & .00 & .00 & .48 & .64 & .00 & -.04 & -.02 & .03 & -.14 & .41 & -.59 \\
\hline Var6 & .00 & .00 & .00 & .00 & .00 & .84 & .00 & .03 & .08 & -.21 & -.28 & .54 \\
\hline
\end{tabular}

Note: For FA and PCA residual structures, residual covariances are shown on and below diagonal; covariances rescaled to correlation metric above diagonal (and italicized to ease comparisons). 
Table 6

Common Factor and Principal Component Solutions for One-Dimensional Batteries Consistent with PCA Representation

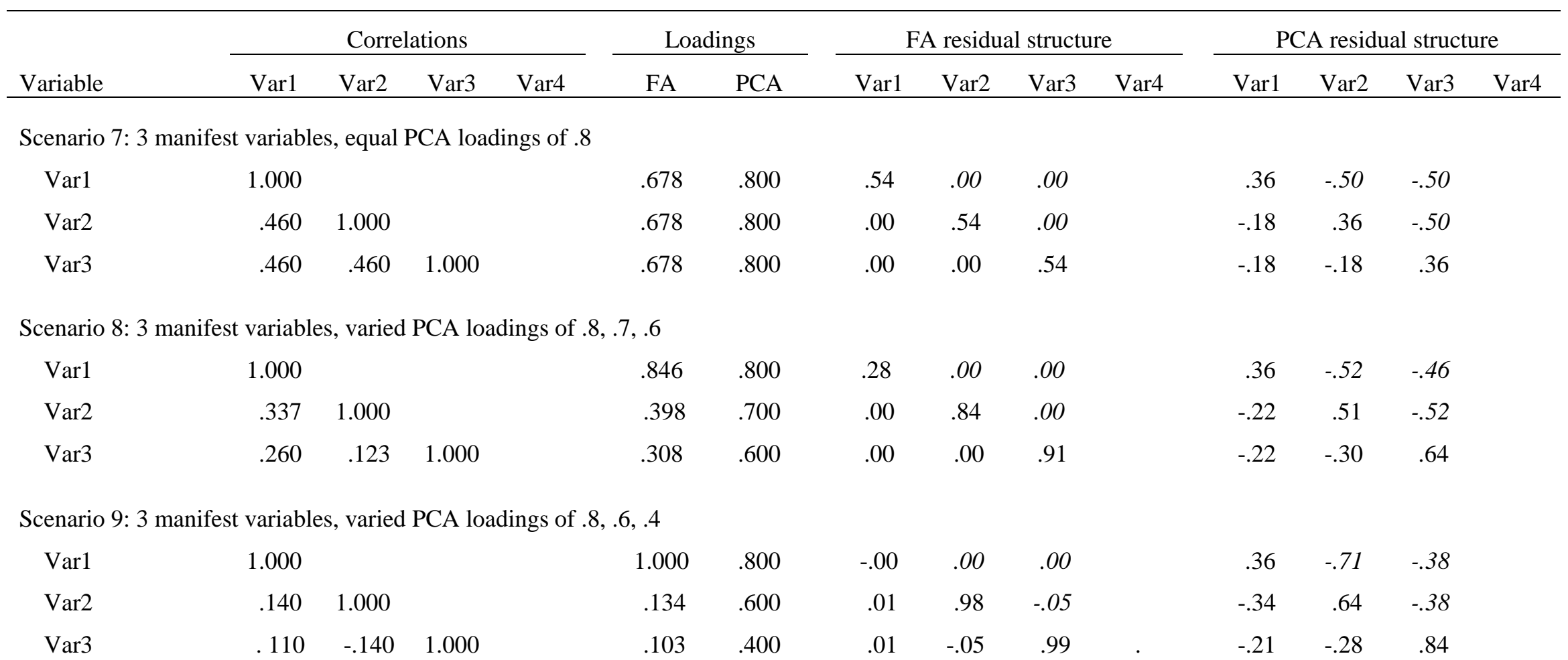

Scenario 10: 4 manifest variables, equal PCA loadings of .8, discarded component eigenvalues of $0.48,0.48,0.48$

\begin{tabular}{|c|c|c|c|c|c|c|c|c|c|c|c|c|c|c|}
\hline Var1 & 1.000 & & & & .721 & .800 & .48 & .00 & .00 & .00 & .36 & -.33 & -.33 & -.33 \\
\hline Var2 & .520 & 1.000 & & & .721 & .800 & .00 & .48 & .00 & .00 & -.12 & .36 & -.33 & -.33 \\
\hline Var3 & .520 & .520 & 1.000 & & .721 & .800 & .00 & .00 & .48 & .00 & -.12 & -.12 & .36 & -.33 \\
\hline Var4 & .520 & .520 & .520 & 1.000 & .721 & .800 & .00 & .00 & .00 & .48 & -.12 & -.12 & -.12 & .36 \\
\hline
\end{tabular}


Table 6 (continued)

\begin{tabular}{|c|c|c|c|c|c|c|c|c|c|c|c|c|c|c|}
\hline \multirow[b]{2}{*}{ Variable } & \multicolumn{4}{|c|}{ Correlations } & \multicolumn{2}{|c|}{ Loadings } & \multicolumn{4}{|c|}{ FA residual structure } & \multicolumn{4}{|c|}{ PCA residual structure } \\
\hline & Var1 & Var2 & Var3 & Var4 & FA & PCA & Var1 & Var2 & Var3 & Var4 & Var1 & Var2 & Var3 & Var4 \\
\hline \multicolumn{15}{|c|}{ Scenario 11: 4 manifest variables, equal PCA loadings of .8 , discarded component eigenvalues of $0.72,0.48,0.24$} \\
\hline Var1 & 1.000 & & & & .721 & .800 & .48 & .25 & .00 & -.25 & .36 & .00 & -.33 & -.67 \\
\hline Var2 & .640 & 1.000 & & & .721 & .800 & .12 & .48 & -.25 & .00 & .00 & .36 & -.67 & -.33 \\
\hline Var3 & .520 & .400 & 1.000 & & .721 & .800 & .00 & -.12 & .48 & .25 & -.12 & -.24 & .36 & .00 \\
\hline Var4 & .400 & .520 & .640 & 1.000 & .721 & .800 & -.12 & .00 & .12 & .48 & -.24 & -.12 & .00 & .36 \\
\hline
\end{tabular}

Scenario 12: 4 manifest variables, equal PCA loadings of .8, discarded component eigenvalues of 0.80, 0.32, 0.32

\begin{tabular}{|c|c|c|c|c|c|c|c|c|c|c|c|c|c|c|}
\hline Var1 & 1.000 & & & & .721 & .800 & .48 & .33 & -.17 & -.17 & .36 & .11 & -.56 & -.56 \\
\hline Var2 & .680 & 1.000 & & & .721 & .800 & .16 & .48 & -.17 & -.17 & .04 & .36 & -.56 & -.56 \\
\hline Var3 & .440 & .440 & 1.000 & & .721 & .800 & -.08 & -.08 & .48 & .33 & -.20 & -.20 & .36 & .11 \\
\hline Var4 & .440 & .440 & .680 & 1.000 & .721 & .800 & -.08 & -.08 & .16 & .48 & -.20 & -.20 & .04 & 36 \\
\hline
\end{tabular}

Scenario 13: 4 manifest variables, varied PCA loadings, discarded component eigenvalues of 0.82, 0.56, 0.34

\begin{tabular}{|c|c|c|c|c|c|c|c|c|c|c|c|c|c|c|}
\hline Var1 & 1.000 & & & & .711 & .800 & .49 & .05 & -.15 & .13 & .36 & -.26 & -.57 & -.17 \\
\hline Var2 & .547 & 1.000 & & & .736 & .800 & .02 & .46 & .11 & -.20 & -.09 & .36 & -.17 & -.57 \\
\hline Var3 & .435 & .578 & 1.000 & & .718 & .800 & -.08 & .05 & .48 & .06 & -.21 & -.06 & .36 & -.26 \\
\hline Var4 & .398 & .206 & .356 & 1.000 & .442 & 600 & .08 & -.12 & .04 & .80 & -.08 & -.27 & -.12 & .64 \\
\hline
\end{tabular}

Scenario 14: 4 manifest variables, varied PCA loadings, discarded component eigenvalues of 0.92, 0.46, 0.34

\begin{tabular}{|c|c|c|c|c|c|c|c|c|c|c|c|c|c|c|}
\hline Var1 & 1.000 & & & & .739 & .800 & .45 & .22 & -.08 & -.18 & .36 & .02 & -.46 & -.56 \\
\hline Var2 & .646 & 1.000 & & & .734 & .800 & .10 & .46 & -.14 &.-10 & .01 & .36 & -.56 & -.46 \\
\hline Var3 & .475 & .439 & 1.000 & & .695 & .800 & -.04 & -.07 & .52 & .28 & -.16 & -.20 & .36 & .02 \\
\hline Var4 & .212 & .260 & .488 & 1.000 & .438 & .600 & -.11 & -.06 & .18 & .81 & -.27 & -.22 & .01 & .64 \\
\hline
\end{tabular}

Note: For residual structures, residual covariances are shown on and below diagonal; covariances rescaled to correlation metric above diagonal (and italicized to ease comparisons). 
Table 7

Maximal Correlation between Two Oblique Principal Components as a Function of Number of Manifest Indicators per Component and Indicator Loading

\begin{tabular}{ccccccc}
\hline \multirow{2}{*}{$\begin{array}{c}\text { Component } \\
\text { loadings }\end{array}$} & \multicolumn{6}{c}{ Number of indicators per component } \\
\cline { 2 - 7 } & 3 & 4 & 5 & 6 & 8 & 10 \\
\hline 6 & .05 & .30 & .44 & .53 & .65 & .72 \\
.7 & .31 & .48 & .59 & .65 & .74 & .79 \\
.8 & .47 & .60 & .68 & .73 & .80 & .84 \\
.9 & .58 & .69 & .75 & .79 & .84 & .87 \\
\hline
\end{tabular}

